OPEN ACCESS

Citation: Matteo Boldrini (2020) II radicamento territoriale dei candidati nei collegi uninominali alle elezioni politiche del 2018. Quaderni dell'Osservatorio elettorale - Italian Journal of Electoral Studies, Research Notes, 1. doi: 10.36253/qoe-9820

Received: November 3, 2020

Accepted: December 20, 2019

Published: December 23, 2020

Copyright: $\odot 2020$ MatteoBoldrini. This is an open access, peer-reviewed article published by Firenze University Press (http://www.fupress.com/qoe) and distributed under the terms of the Creative Commons Attribution License, which permits unrestricted use, distribution, and reproduction in any medium, provided the original author and source are credited.

Data Availability Statement: All relevant data are within the paper and its Supporting Information files.

Competing Interests: The Author(s) declare(s) no conflict of interest.

\section{Il radicamento territoriale dei candidati nei collegi uninominali alle elezioni politiche del 2018}

\author{
MATteo BoldRini \\ Department of Social and Political Sciences (DSPS), University of Florence \\ Centre de recherches politiques de la Sorbonne, Université Paris 1 Panthéon-Sorbonne \\ E-mail: matteo.boldrini@unifi.it
}

\begin{abstract}
Among the attributes of political candidates, localness represents an aspect relatively uncovered by the core of the literature on party politics. There are, however, different scholars that pinpointed to the utility of this aspect in understanding political elites' circulation. In line with this assumption, the article aims to map the degree of localness of single-member constituencies' candidates in the 2018 Italian general elections. More specifically, the analysis focuses on the candidates' localness by highlight similarities and differences among party-candidates and their geographical distribution. In doing so, the analysis is based on an original dataset; the localness of candidates has been calculated through a localness index. The article is organized as follows. It starts from a literature review on localness. Drawing on this theoretical introduction, I identify the logic behind the research question. In the next section, the rationale for the selection of the different cases analysed is provided. On this ground, the analysis proceeds with a synthetic overview of the context of reference. In the last part of the analysis, the index of localness is defined and applied to the dataset. The main conclusion is that, although there is a strong diffusion of local candidates, there are significant differences among parties, with a higher localness level of the League and the Democratic Party than Forza Italia and the Five Star Movement, especially in the Northern and Southern's regions.
\end{abstract}

Keywords. Candidates, party politics, localness, elections, Italy.

\section{INTRODUZIONE}

Lo studio della classe politica rappresenta con ogni probabilità uno degli argomenti classici di analisi da parte delle scienze politiche (Sola 2003). In particolar modo, uno degli aspetti maggiormente discussi è probabilmente quello relativo alle caratteristiche dei componenti della classe politica, come ad esempio, il genere, il titolo di studio, l'appartenenza professionale. All'interno di questo filone di studi, uno degli ambiti che in passato non è stato sempre adeguatamente approfondito è quello relativo al radicamento territoriale. Il rapporto che lega il candidato con il collegio o la circoscrizione di appartenenza non è stato spesso oggetto di indagini sistematiche da parte degli scienziati 
sociali. Tuttavia, il tema ha assunto progressivamente una maggiore rilevanza sia nel dibattito pubblico, sia in quello scientifico. Da un lato, infatti, sempre più spesso le forze politiche sembrano impegnate nel cercare di presentare $\mathrm{i}$ propri candidati come fortemente legati al territorio di candidatura; dall'altro, le ricerche degli scienziati sociali hanno evidenziato la rilevanza del radicamento territoriale nell'orientare il comportamento degli elettori e nel modificare le strategie dei partiti e degli stessi esponenti della classe politica (Tavits 2010; Shugart, Valdini e Souminen 2005).

Il radicamento territoriale dei candidati rappresenta dunque un ambito relativamente poco studiato da parte della letteratura. Tuttavia, un suo esame più approfondito potrebbe essere utile al fine di meglio comprendere le dinamiche di trasformazione dei partiti, del ceto politico locale e dei legami che li ricollegano alla società e agli elettori.

L'obiettivo di questa ricerca è quello di approfondire questo aspetto, andando ad indagare quale sia stato il radicamento territoriale dei candidati presentati dalle principali forze politiche alle ultime elezioni politiche italiane. La finalità della ricerca, concentratasi sull'esame di un singolo caso di studio, è dunque allo stesso tempo sia descrittiva che esplorativa. L'obiettivo che ci si propone non è infatti quello di formulare considerazioni generalizzabili, ma quello di cercare di descrivere dettagliatamente il fenomeno del radicamento territoriale dei candidati, contribuendo allo stesso tempo all'apertura di nuove piste di ricerca e fornenedo il materiale per la formulazione di ulteriori ipotesi da testare in futuro. La natura descrittiva, insieme alle difficoltà che si incontrano nella costruzione del dato e alla complessità del fenomeno in oggetto, giustificano quindi la scelta di concentrarsi solamente su un unico caso di studio (Yin 2009). La scelta delle ultime elezioni politiche italiane è stata dettata poi dalla disponibilità di dati a disposizione e dalla presenza di alcune particolarità che rendono interessante il suo esame. Le elezioni politiche del 2018 hanno rappresentato, all'interno dello scenario politico italiano, una forte discontinuità sotto numerosi punti di vista (Paparo e Emanuele 2018). Tra di essi, uno dei più significativi è senza dubbio il ritorno, dopo quasi vent'anni, ad una legge elettorale mista contenente una parte di collegi uninominali (Chiaramonte e D'Alimonte 2018). Il ritorno dei collegi, con la loro competizione maggioritaria e la maggiore visibilità associata ai singoli candidati, spinge ad interrogarsi su quali siano le caratteristiche del personale politico presentato dai vari partiti all'interno dei collegi stessi.

Considerati questi aspetti, ci proponiamo quindi di esaminare il radicamento territoriale dei candidati attra- verso l'utilizzo di un indice di radicamento territoriale elaborato dalla letteratura e un dataset originale relativo ai candidati delle tre principali forze politiche (centrodestra, centrosinistra, Movimento Cinque Stelle) presentatisi nei 241 collegi elettorali uninominali (tutti i collegi previsti dalla legge 165/2017 ad eccezione del collegio della Valle d'Aosta) nel 2018. Il radicamento territoriale a cui si è scelto di fare riferimento è quello relativo agli attributi personali dei candidati, considerando come radicati quei candidati che presentano una biografia personale (legata ad indicatori come il luogo di nascita e le precedenti esperienze amministrative) fortemente legata al collegio di candidatura. Oltre al radicamento territoriale e all'appartenenza politica si è scelto di considerare anche l'area geografica di candidatura, in maniera tale da cercare di comprendere se esista (e nel caso quale sia) l'area geografica del Paese in cui le forze politiche concentrano i candidati maggiormente radicati rispetto a quelli "paracadutati".

Per rispondere alla nostra domanda di ricerca, nella prima parte dell'articolo verranno esaminati i vari studi effettuati sul tema del radicamento territoriale della classe politica italiana, quali sono state le principali linee di ricerca e quali siano le principali linee di tendenza che tali ricerche hanno segnalato sulla classe politica, avendo così un quadro di riferimento che ci permetta di formulare delle ipotesi su come ci si aspetta che i candidati siano (o nel caso non siano) radicati. Nella seconda parte invece verrà presentata l'evoluzione del radicamento territoriale nel caso italiano, i metodi e le tecniche con cui è stato calcolato l'indice di radicamento territoriale, e il metodo da noi adottato per calcolarlo e i motivi di tale scelta. Successivamente, nei due paragrafi conclusivi, si procederà alla presentazione e all'analisi dei dati e alle conclusioni.

\section{INQUADRAMENTO TEORICO E ASSUNTI DELLA RICERCA}

Il profilo dei candidati rappresenta una delle modalità tradizionali mediante la quale si articola l'offerta politica presentata dai partiti agli elettori. L'insieme delle caratteristiche politiche, ma anche personali e biografiche, costituisce infatti la modalità più immediata con cui le forze politiche esercitano una forma di rappresentanza descrittiva (Pitkin 1967), provando a tessere legami progressivamente più stretti con il proprio elettorato e con i propri gruppi sociali di riferimento (Philips 1995). Tratti come la provenienza di classe o professionale, il genere e l'appartenenza etnica, hanno rappresentato a lungo un oggetto di studio privilegiato all'interno delle 
indagini socio-politologiche. Accanto a questi, in anni relativamente recenti, si è assistito ad un crescente interesse da parte degli studiosi sui tratti "locali" del personale politico (Childs e Cowley 2011; Collignon-Delmar e Sajuria 2018). Numerose ricerche hanno infatti sottolineato come un profilo politico fortemente radicato possa rappresentare una dimensione estremamente rilevante nell'analisi della classe politica, sia dal punto di vista della sua attività all'interno delle assemblee legislative, sia da quello della competizione elettorale.

Gli sforzi degli studiosi si sono concentrati principalmente su tre differenti filoni di ricerca, relativi all'attività parlamentare degli eletti, al vantaggio competitivo di candidati maggiormente radicati ed infine al rapporto tra radicamento territoriale e sistemi elettorali. Per quanto riguarda il primo filone di ricerca, parte della letteratura ha osservato come il radicamento territoriale rappresenti una variabile significativa nell'orientare il comportamento degli eletti e l'attività all'interno delle assemblee legislative. È stato infatti notato che i parlamentari aventi un profilo più locale e maggiormente radicato sul territorio siano tendenzialmente più propensi a votare in dissenso rispetto al partito e al gruppo di appartenenza e più attenti ad un'azione politica rivolta verso gli interessi del proprio collegio di elezione (Marangoni e Tronconi 2011; Crisp e Desposato 2004; Tavits 2010). Il secondo filone di ricerca ha poi sottolineato come un solido legame con il territorio possa rappresentare una risorsa importante all'interno della competizione elettorale, con candidati fortemente legati alla propria circoscrizione di elezione che risultano essere maggiormente competitivi rispetto a coloro che non lo sono (Putnam 1976; Marsh 1987; Shugart, Valdini e Souminen 2005; Tavits 2010). Infine, parte degli studiosi ha messo in relazione gli incentivi forniti dai sistemi elettorali alla presenza di candidati più radicati. Alla consolidata letteratura sul rapporto tra radicamento e collegio uninominale, (Arzheimer e Evans 2012; Fenno 1978; Fiva e Smith 2017), in anni recenti, si sono aggiunte numerose indagini relative alla relazione esistente tra sistemi proporzionali e radicamento territoriale. In particolare, è stato evidenziato come un sistema elettorale, con circoscrizioni piccole e con una competizione più aperta, favorisca la presenza di parlamentari con un alto grado di radicamento territoriale (Shugart, Valdini e Souminen 2005). Infatti, all'aumentare dell'apertura della competizione e al diminuire della magnitudine della circoscrizione, un maggiore radicamento territoriale fornisce ai candidati un significativo vantaggio nell'intercettare voti personali, rendendoli quindi maggiormente competitivi verso eventuali concorrenti, sia del proprio partito sia di forze politiche avversarie. È stato ipotizzato che attributi personali indicanti un maggior radicamento territoriale, come la coincidenza tra luogo di nascita e quello di candidatura e l'aver svolto altre attività politiche a livello locale nel proprio collegio, possano essere interpretate da parte degli elettori come una maggiore attenzione ai problemi del territorio operando sostanzialmente come una "scorciatoia" utile ad individuare quali candidati possano meglio difendere gli interessi locali, svolgendo quindi una funzione che è stata definita analoga al "constituency service" (Putnam 1976; Marsh 1987; Shugart, Valdini e Souminen 2005; Tavits 2010).

Le ricerche hanno dunque evidenziato come il radicamento territoriale possa rappresentare una importante risorsa per i partiti e la classe politica. Tuttavia, la gran parte degli studi sul tema si sono concentrati sulla classe politica eletta, cioè sugli esponenti usciti vittoriosi dalla competizione elettorale e quindi già facenti parte della classe parlamentare, tralasciando di esaminare i tratti dei candidati usciti sconfitti dalle elezioni. Ciò è dovuto principalmente alla maggiore facilità di reperimento dei dati, in quanto difficilmente vengono presentati quelli relativi ai candidati non risultati eletti (Tavits 2010). Tuttavia, per poter comprendere meglio il ruolo del radicamento territoriale nello scenario politico contemporaneo, diviene necessario esaminare l'insieme dei candidati presentati alle elezioni. In questa maniera, infatti, non solo si è in grado di meglio comprendere quanto effettivamente sia radicato l'insieme del personale politico, ma anche quanto il radicamento stesso rappresenti una risorsa più o meno significativa per le forze politiche.

Inoltre, il radicamento territoriale viene solitamente rappresentato come un attributo che può essere o meno posseduto dai vari esponenti della classe politica. Esso, pur rappresentando un utile strumento in grado di sintetizzare rapidamente il grado di radicamento dei candidati, limita la portata della comprensione del fenomeno. Se, come infatti abbiamo visto, il ruolo degli attributi relativi al radicamento territoriale è quello di svolgere per gli elettori una funzione di indicazione di quali siano i candidati maggiormente attenti alle tematiche locali, ci si può aspettare che esistano significative sfumature all'interno delle differenti forme di radicamento e che gli elettori reagiscano in maniera diversa a livelli più $o$ meno alti di radicamento. È infatti ragionevole supporre che un candidato, nato e con alle spalle incarichi amministrativi all'interno del proprio collegio, possa essere considerato dagli elettori come maggiormente attento ai problemi del collegio di uno che vi è solo nato e, conseguentemente, meno attento di un candidato che in aggiunta ricopre un qualche incarico amministrativo al momento della candidatura. Sostanzialmente dunque, il radicamento territoriale si presta ad essere meglio consi- 
derato come una caratteristica con più gradi, invece che come un attributo che può essere posseduto o meno.

All'interno di questo articolo si è scelto di adottare una prospettiva che cercasse di approfondire questi due differenti aspetti che in passato hanno ricevuto meno attenzione da parte degli scienziati sociali. La ricerca si è proposta dunque di indagare quale sia stata, dal punto di vista del radicamento territoriale, l'offerta elettorale presentata dalle principali forze politiche alle elezioni italiane del 2018, evidenziando in che maniera il ritorno al collegio uninominale abbia spinto verso la selezione di candidati fortemente radicati sul territorio, concentrandosi poi sulla loro distribuzione lungo il territorio nazionale e sulle differenze che intercorrono tra le diverse forze politiche. L'esame delle differenze nel radicamento tra i vari partiti politici è infatti un utile strumento volto a comprendere meglio quali siano le dinamiche di funzionamento dei partiti e della democrazia italiana. In primo luogo, esso ci aiuta a rilevare quali siano le dinamiche di trasformazione e di circolazione della classe politica e quali siano i percorsi principali attraverso i quali avviene il suo reclutamento. Secondariamente, l'analisi del radicamento ci permette di esaminare, chiaramente in via preliminare e suscettibile di ulteriori approfondimenti, in che maniera le varie forze politiche diano spazio alla rappresentanza delle istanze locali all'interno dello scenario nazionale. Infine, esso può rappresentare un utile strumento per la comprensione delle dinamiche di mutamento dei partiti. Seguendo parte della letteratura sul cambiamento dei partiti politici (Harmel e Janda 1994), il radicamento dei candidati può infatti essere considerato come parte della dimensione organizzativa e strutturale dei partiti, ed una sua modifica nel corso del tempo può quindi aiutare nell'interpretazione dei mutamenti dei vari partiti politici.

\section{LA SELEZIONE DEL CASO}

Date le finalità descrittive ed esplorative che ci siamo proposti, l'esame di un caso di studio è stata la modalità di ricerca che è sembrata più appropriata. I casi di studio rappresentano infatti uno dei metodi che meglio si prestano all'esecuzione di ricerche aventi questo tipo di finalità (Yin 2009; Yin 2013). A queste motivazioni si sono aggiunte poi alcune considerazioni relative alle caratteristiche dell'oggetto di studio e alle modalità di reperimento dei dati. L'assenza di studi sistematici sul tema e la scelta di utilizzare una particolare forma di indice di radicamento ha infatti richiesto una raccolta dei dati estremamente dettagliata e puntuale, non effettuabile attraverso archivi o database organizza- ti. Questa necessità ci ha spinto dunque per la selezione di un metodo di ricerca il più possibile dettagliato, come appunto quello dello studio di caso, volendo in questo modo svolgere una prima analisi utile per future comparazioni o approfondimenti. La scelta di concentrarsi sulle elezioni politiche italiane del 2018 è stata anzitutto motivata da un aspetto pragmatico legato alla maggiore facilità di reperimento dei dati. La complessità nella costruzione del dato ci ha infatti spinto a selezionare un'elezione vicina nel tempo, in modo tale da permettere una maggiore precisione ed affidabilità.

Inoltre, queste elezioni presentano alcune caratteristiche che le rendono particolarmente interessanti. In primo luogo, esse vedono il ritorno di una legge elettorale mista proporzionale e maggioritaria dopo quasi vent'anni dall'abrogazione della legge Mattarella nel 2001. Il ritorno dei collegi ci consente quindi di andare ad indagare in che maniera si sia sviluppato il radicamento territoriale dei candidati che vi si sono presentati. Secondariamente, le elezioni del 2018 presentano delle caratteristiche interessanti dal punto di vista del cambiamento del sistema politico. Infatti, l'offerta politica risulta estremamente differente rispetto sia all'ultima elezione con il collegio uninominale, con un inedito sistema politico di natura tripolare, sia soprattutto rispetto alle elezioni politiche del 2013. I cambiamenti più interessanti hanno interessato le tre principali forze politiche del Paese. All'interno della coalizione di centrodestra, Forza Italia è stata gradualmente sostituita dalla Lega nel ruolo di partner principale della coalizione, che per la prima volta ha adottato il profilo di un partito "nazionale" cercando di raccogliere consensi in tutto il Paese (Emanuale e Paparo 2018; Passarelli e Tuorto 2018). Nel centrosinistra invece, il Partito Democratico, alleato con le due liste di Civica Popolare e Insieme, si è presentato alle elezioni sotto la segreteria di Matteo Renzi con un partito significativamente differente rispetto a quello delle politiche del 2013 (Ventura 2018). Infine, il Movimento Cinque Stelle, dopo il grande risultato ottenuto nel 2013 (Chiaramonte e De Sio 2014), si è misurato con l'introduzione dei collegi uninominali alla ricerca di una conferma del risultato di cinque anni prima e di un radicamento dei propri consensi all'interno del Paese. Com'è logico immaginarsi, il processo che porta alla definizione delle candidature è chiaramente un processo complesso in cui a fattori di competizione intra-partitica si sommano fattori relativi alla competizione tra partiti, alle strategie da essi adottate e agli incentivi istituzionali offerti dalla legge elettorale e dal sistema politico (Marangoni e Tronconi 2009). La differente struttura delle opportunità, quindi, potrebbe aver spinto le diverse forze politiche verso una rivalutazione del radicamento 
territoriale dei candidati, considerando personalità fortemente radicate all'interno del contesto sociale come maggiormente competitive in fase elettorale.

I candidati oggetto dell'analisi sono stati quelli relativi alle tre principali forze politiche presentatesi a questa elezione, le due coalizioni di centrodestra e centrosinistra e il Movimento Cinque Stelle, non considerando i candidati presentati dalle forze più piccole. I motivi legati a questa scelta sono collegati alla maggiore reperibilità dei dati, difficilmente rilevabili per i candidati delle forze più piccole e con poche possibilità di vincere il collegio e alla maggiore rilevanza di queste tre forze politiche che complessivamente raccolgono la quasi totalità dei seggi all'interno dei collegi uninominali. In particolare, la scelta di considerare il M5S è stata dettata dalla rilevanza ricoperta dal Movimento all'interno del sistema politico italiano, sebbene, come vedremo, in virtù dei suoi tratti, una lettura del radicamento territoriale come da noi proposto diviene più complessa. Caratteristiche principali del Movimento sono state infatti, almeno fino alle elezioni del 2018, la presenza di un limite massimo di due mandati esercitabili nel corso della propria carriera politica (conteggiando all'interno del limite anche gli eventuali mandati amministrativi effettuati all'interno di enti locali e regionali) e un limite alla possibilità di candidarsi per altri incarichi durante un mandato ricoperto in altri enti. Appare dunque evidente che limiti così concepiti rendono particolarmente difficoltoso lo sviluppo di un radicamento territoriale rilevabile attraverso i metodi utilizzati all'interno della ricerca.

\subsection{Il radicamento territoriale nel caso italiano}

Il caso italiano, alla luce dei numerosi cambiamenti del suo sistema politico ed istituzionale, rappresenta probabilmente uno dei casi più emblematici per l'esame del radicamento territoriale della classe politica. Paese contraddistinto storicamente per una forte presenza dei partiti nella società, si caratterizzava però per un legame tra parlamentari e territorio di appartenenza particolarmente debole. Le ricerche degli scienziati sociali hanno infatti evidenziato come, a partire dalla Prima Repubbli$\mathrm{ca}$, vi fosse un legame sostanzialmente poco strutturato tra l'eletto e la propria circoscrizione di elezione rispetto agli altri Paesi europei (Tronconi e Verzichelli 2007). I grandi soggetti politici che animavano la vita politica italiana, sebbene fossero dotati di una capillare presenza all'interno della società e di un forte ancoraggio all'interno delle amministrazioni locali, tendevano ad intrepretare la rappresentanza in chiave più "nazionale" che non "locale", con una gestione delle candidature e della rappresentanza parlamentare rigidamente controllata al livello centrale da parte dei gruppi dirigenti dei partiti politici (Cotta 1979). A questi fattori si aggiungevano poi gli scarsi incentivi istituzionali offerti dal sistema, primo tra tutti la legge elettorale di tipo proporzionale, che non favoriva le condizioni per lo sviluppo di un forte legame tra il territorio e l'eletto (Marangoni e Tronconi 2009). Questa tensione verso una rappresentanza di tipo nazionale veniva però leggermente affievolita dall'ampio utilizzo del voto di preferenza da parte degli elettori di alcune forze politiche (soprattutto in determinate aree geografiche del Paese). L'utilizzo delle preferenze era infatti molto più diffuso nelle regioni del Sud Italia rispetto a quelle settentrionali e tra gli esponenti della Democrazia Cristiana (Diamanti 2003; Parisi e Pasquino 1977) che, rispetto al Partito Comunista, univa ai tratti tipici del partito di massa una significativa presenza di voto personale e clientelare (Cotta 1979).

La fine della Prima Repubblica e le modifiche al sistema dei partiti degli anni Novanta non sembrano aver intaccato significativamente questa caratteristica del sistema politico italiano.

Gli studi effettuati sulla classe politica della Seconda Repubblica hanno infatti confermato la tendenza ad una cronica debolezza del rapporto tra eletti e territorio. Nonostante l'introduzione, nella legge elettorale del 1993, di una formula mista maggioritaria e proporzionale (D'Alimonte e Chiaramonte 1994), il dato relativo alla territorializzazione dei candidati, in particolar modo di quelli nei collegi uninominali, rimane sostanzialmente basso (Fabbrini 1996), sebbene con gradi differenti tra le varie forze politiche e le varie zone d'Italia. Una maggiore attenzione alla dimensione territoriale dei candidati era presente tra i candidati del Partito Democratico della Sinistra e del Partito Popolare rispetto a partiti come Forza Italia e ai partiti della destra, e tra i candidati meridionali rispetto a quelli settentrionali (Mattina e Tonarelli, 1996). Ricapitolando, il meccanismo che guidava la scelta delle candidature seguiva principalmente una logica di tipo "nazionale" in cui la selezione dei candidati all'interno dei collegi uninominali seguiva sostanzialmente una logica di spartizione delle candidature e dei collegi "sicuri" adottata a livello centrale tra i vari partner della coalizione (Verzichelli 1996, p. 735). La riforma elettorale del 2005, con l'introduzione del proporzionale con premio di maggioranza, finì per interrompere bruscamente i tentativi di adattamento del sistema politico all'introduzione dei collegi (Verzichelli e Legnante 2005; Tronconi e Verzichelli 2007). Come abbiamo anticipato, non tutte le forze politiche presentavano uno stesso grado di radicamento. Le ricerche hanno infatti evidenziato come i partiti molto piccoli oppure dotati di una struttura organizzativa più "leggera", come ad esempio Forza 
Italia, presentavano tradizionalmente un più basso legame tra la classe parlamentare e il territorio di appartenenza; viceversa, partiti con un solido insediamento territoriale oppure localizzati in un preciso contesto territoriale presentavano tassi di radicamento più alti (D'Alimonte e Bartolini 2002; Tronconi e Verzichelli 2007; Verzichelli 2006). La Lega Nord, in particolar modo, sia per la sua natura di partito regionale (e quindi presente solamente in alcune zone del paese) sia per la sua forte strutturazione a livello locale, presentava tassi di radicamento territoriale sistematicamente più alti rispetto a quelli degli altri partiti (Verzichelli 2005). Ad eccezione della Lega dunque, il dato generale è che le varie forze politiche si siano dimostrate scarsamente attente al radicamento territoriale dei propri candidati, preferendovi una logica di reclutamento molto più ancorata alle dinamiche della competizione politica nazionale.

Sulla base di quanto detto fino ad ora dunque, è possibile formulare alcune aspettative sui risultati dell'analisi. In primo luogo, ci si aspetta che ci sia una differenza significativa tra i partiti "tradizionali" e il Movimento Cinque Stelle: i primi dotati di un maggiore radicamento e il secondo invece più ancorato a dinamiche nazionali. Secondariamente, ci si aspetta che ci siano delle differenze tra quelle forze politiche storicamente dotate di un maggiore ancoraggio territoriale, Lega e centrosinistra, e le altre. Infine, è ragionevole aspettarsi che vi siano bacini di reclutamento territoriale differenti per le varie forze politiche, con la coalizione di centrodestra maggiormente radicata nelle regioni settentrionali e quella di centro-sinistra nelle regioni centrali del Paese.

\section{L'INDICE DI RADICAMENTO E IL DATASET}

Le modalità con cui in passato gli studiosi hanno cercato di rilevare il radicamento territoriale sono state numerose. All'interno delle ricerche che considerano il radicamento come un attributo del candidato e mirano quindi a rilevarlo attraverso le sue caratteristiche biografiche, i principali indicatori tradizionalmente usati dagli scienziati politici sono stati la coincidenza tra il luogo di nascita del candidato (o in certi casi il luogo di residenza) e l'aver ricoperto in passato degli incarichi amministrativi in enti locali presenti all'interno del collegio o della circoscrizione di elezione. I due indicatori vengono solitamente operazionalizzati come variabili dicotomiche, considerando quindi come "locale" (e quindi radicato territorialmente) il parlamentare candidato nella circoscrizione o nel collegio in cui è nato o in cui ha ricoperto incarichi amministrativi a livello locale (Shugart, Valdini e Souminen 2005; Tavits 2009, 2010). Viceversa, un candidato è considerato nel profilo "nazionale" se invece non possiede gli attributi sopra menzionati. Questa operazionalizzazione, sebbene rappresenti uno strumento volto ad una comprensione rapida del rapporto tra la classe politica ed il territorio, è stata ritenuta da una parte della letteratura non completamente soddisfacente, in quanto eccessivamente riduttiva e portata ad errate rappresentazioni del radicamento di alcuni candidati. Un esempio tipico è rappresentato da quei candidati che, pur essendo nati in una determinata città, l'abbandonano per poi venire lì candidati molti anni più tardi e risultando quindi ai sensi dell'indicatore come "radicati". Un limite ulteriore è poi rappresentato dalla sua dimensione strettamente dicotomica, che, rappresentando il radicamento come un attributo che può essere o meno posseduto, tende a rendere eccessivamente omogenee situazioni estremamente differenti. I candidati possono infatti presentare sfumature di radicamento estremamente differenti tra loro e gli stessi elettori possono quindi essere portati a reagire in maniera differente a queste differenti sfumature. I due autori Marangoni e Tronconi (2011) hanno cercato di risolvere parzialmente queste problematiche, attraverso un originale indice di radicamento territoriale che si propone di misurare il radicamento dei parlamentari non tanto attraverso una sua rappresentazione dicotomica, ma mediante l'utilizzo di un indice suscettibile di assumere diverse gradazioni, considerando contemporaneamente come indicatori del radicamento sia i fattori relativi al luogo di nascita che quelli relativi all'esperienza amministrativa (Marangoni e Tronconi 2011, p. 9). L'obiettivo è quello di rappresentare il radicamento territoriale non tanto come un attributo che può essere posseduto o meno da parte dei candidati, quanto piuttosto come una caratteristica dotata di diverse sfumature. Pur nella consapevolezza della maggiore complessità della realtà rispetto a quanto rappresentabile attraverso la semplice operazionalizzazione delle variabili, un approccio come quello proposto dai due autori è stato ritenuto più adatto a rappresentare quale sia il radicamento territoriale dei candidati alle elezioni politiche del 2018. L'indice, a nostro giudizio, permette di superare almeno parzialmente alcuni dei limiti sopra esposti. L'utilizzo di una variabile con varie gradazioni conferisce alla dimensione del radicamento territoriale un maggiore livello di profondità, dando una rappresentazione più realistica del fenomeno e permettendo ampi spazi di comparazione e di analisi. Esso permette infatti di rappresentare le varie differenze che possono intercorrere tra gli stessi candidati radicati e misurare l'effetto delle diverse sfumature di radicamento.

Si è dunque scelto di utilizzare l'index of localness elaborato da Marangoni e Tronconi (2011), adattato alle esigenze della ricerca sui candidati nei collegi uninominali. 
L'indice utilizzato può assumere valori compresi tra 1 e 4 in base all'eventuale presenza del comune (tutto o in parte) di nascita e/o di un ente locale (tutto o in parte) in cui il candidato ha ricoperto precedenti incarichi amministrativi all'interno del collegio di candidatura ${ }^{1}$, e all'eventuale candidatura intercorsa mentre ancora occupava l'ufficio all'interno dell'ente locale ${ }^{2}$.

All'interno della Tabella 1 sono rappresentati sinteticamente gli indicatori utilizzati nella costruzione dell'indice.

Seguendo quanto detto, abbiamo quindi considerato come avente un profilo "nazionale" (corrispondente ad un valore 1) il candidato che risulta essere nato all'interno di un comune non incluso (neanche in misura parziale) nel collegio uninominale in cui è stato candidato alle elezioni e che non ha mai ricoperto alcun incarico amministrativo in un ente locale presente all'interno del collegio.

I candidati sono invece stati considerati con un basso grado di radicamento (valore 2) se la loro candidatura è avvenuta in un collegio comprendente il comune di nascita del candidato, oppure comprendente un ente locale in cui hanno ricoperto incarichi amministrativi. Un maggiore grado di radicamento territoriale (valore 3) è stato invece riservato a quei candidati che risultano essere nati in un comune compreso all'interno del collegio uninominale e che hanno in aggiunta anche ricoperto incarichi amministrativi o che, pur non essendo nati all'interno del collegio, ricoprivano qualche incarico amministrativo all'interno del collegio di elezione e la loro candidatura è avvenuta durante il mandato amministrativo. Infine, il massimo grado di radicamento territoriale (valore pari a 4) è riservato a quei candidati nati all'interno del collegio e che vi hanno ricoperto un incarico amministrativo nei 365 giorni precedenti le elezioni.

Come già detto, sono state indagate le caratteristiche dei candidati delle tre principali forze politiche presentatisi nei 231 collegi uninominali in cui è stato diviso il

\footnotetext{
${ }^{1}$ All'interno della dicitura "ente locale" si è scelto di comprendere il comune, la provincia e la Regione, considerando quindi come incarichi in enti locali gli incarichi di consigliere comunali, assessore, sindaco, consigliere provinciale, assessore provinciale, presidente di provincia, consigliere regionale, assessore regionale, presidente di regione. La scelta di considerare tutto l'ente di nascita o di appartenenza oppure una parte di esso ci ha consente di ovviare al problema di collegi che contengono solo parzialmente gli enti locali di riferimento (è il caso di praticamente tutte le grandi città, suddivise in più collegi in base alle sezioni elettorali). Per quanto riguarda l'aver ricoperto incarichi all'interno delle Regioni invece, enti di dimensioni maggiori rispetto ai collegi, esso è stato considerato solo qualora il collegio di candidatura sia, in tutto o in parte, incluse nella regione in cui il candidato ha ricoperto l'incarico. ${ }^{2}$ Come è noto, la legge impone alcuni limiti alla possibilità di candidarsi per gli amministratori locali e regionali. Per ovviare a questo limite si è considerato come "candidato come ancora in carica", se risultava in carica nei 365 giorni precedenti le elezioni.
}

Tabella 1. Indice di radicamento territoriale.

\begin{tabular}{|c|c|c|c|}
\hline \multicolumn{4}{|c|}{ Indice di radicamento territoriale } \\
\hline 1 (profilo nazionale) & 2 (profilo semi-nazionale) & 3 (profilo semi-locale) & 4 (profilo locale) \\
\hline \multirow[t]{2}{*}{$\begin{array}{l}\text { Comune di nascita } \\
\text { esterno al collegio di } \\
\text { candidatura e nessuna } \\
\text { esperienza } \\
\text { amministrativa in enti } \\
\text { inclusi nel collegio }\end{array}$} & $\begin{array}{l}\text { Comune di nascita interno al } \\
\text { collegio di candidatura }\end{array}$ & $\begin{array}{l}\text { Comune di } \text { nascita } \\
\text { interno al collegio di } \\
\text { candidatura } \\
\text { esperienza } \\
\text { amministrativa in enti } \\
\text { locali inclusi nel collegio }\end{array}$ & $\begin{array}{l}\text { Comune di nascita } \\
\text { interno al collegio } \\
\text { di candidatura ed } \\
\text { esperienza } \\
\text { amministrativa in } \\
\text { enti locali inclusi } \\
\text { nel collegio al } \\
\text { momento della } \\
\text { candidatura }\end{array}$ \\
\hline & $\begin{array}{l}\text { Esperienza amministrativa in } \\
\text { enti locali inclusi nel collegio }\end{array}$ & $\begin{array}{l}\text { Esperienza } \\
\text { amministrativa in enti } \\
\text { locali inclusi nel collegio } \\
\text { al momento della } \\
\text { candidatura }\end{array}$ & \\
\hline
\end{tabular}

(Fonte: Elaborazione dell'autore)

Paese (tutti i collegi ad eccezione del collegio della Valle d'Aosta, in virtù delle sue peculiari caratteristiche politiche) per un totale di 693 candidati.

La raccolta dei dati è stata effettuata attraverso l'Anagrafe elettorale degli amministratori locali del Ministero dell'Interno, che raccoglie i principali dati biografici (incluso il luogo di nascita) di tutti gli amministratori locali dal 1982 ad oggi. Per quei candidati che non hanno mai ricoperto alcun tipo di incarico amministrativo, il luogo di nascita è stato identificato mediante accesso ai siti web e alle pagine personali dei candidati presenti sui social network, nonché agli articoli presenti sulla stampa quotidiana e sui giornali online.

Con l'eccezione della Lega nel centrodestra, le coalizioni sono state poi considerate per blocchi, senza quindi scendere nel dettaglio delle singole liste che hanno espresso i vari candidati. Questo da un lato per includere all'interno dell'analisi la Lega, che, come vedremo, esprime un numero considerevole di candidati e che storicamente si è contraddistinta per un forte legame con il territorio; dall'altro per evitare di soffermarsi su liste troppo piccole che, con un numero ridotto di candidati all'interno delle coalizioni, rischierebbero di mostrare valori troppo elevati in un senso o nell'altro e quindi non facilmente confrontabili. Il numero abbastanza esiguo dei candidati delle altre forze politiche che compongono le coalizioni (come ad esempio Civica Popolare e Insieme nel centrosinistra) renderebbe infatti più difficoltosa una loro comparazione ${ }^{3}$.

${ }^{3}$ Se si esclude infatti la Lega, presente con una pattuglia di ben 76 candidati, le altre forze politiche esprimono un numero di candidati particolarmente piccolo. Numeri così bassi, renderebbero più difficoltosa la lettura dei dati, sena aggiungere tantissimo all'analisi comparativa, in quanto spesso presenti con uno o due deputati al massimo per area geografica. Infine, in certi casi, è risultata particolarmente difficoltosa l'assegnazione di un candidato ad una particolare forza politica. Dati 
Venendo infine alle ipotesi che hanno guidato la ricerca, si può facilmente ipotizzare che vi siano delle significative differenze tra i vari partiti. Ci si può aspettare infatti che, nonostante la nazionalizzazione, la Lega presenti un tasso di candidati fortemente radicati più alto rispetto agli altri partiti e tendenzialmente ancora concentrato nelle regioni del centro-nord, tradizionale bacino di reclutamento della classe politica leghista. Specularmente, è ipotizzabile che Forza Italia e gli altri partiti del centrodestra, presentino un radicamento territoriale dei propri candidati complessivamente più basso ma tendenzialmente più alto nelle regioni meridionali del Paese. Per quanto riguarda il Partito Democratico e i suoi alleati, ci si potrebbe aspettare una maggiore percentuale di candidati radicati all'interno dei collegi del centro-Italia, zone di tradizionale radicamento delle forze di centrosinistra. Viceversa, si può invece ipotizzare che i partiti di centrosinistra, per poter meglio competere con le altre forze politiche, cerchino di recuperare margini di consenso attraverso la candidatura di personalità più radicate nel territorio in quelle aree che in passato risultavano più deboli dal punto di vista del sostegno elettorale. Infine, per quanto riguarda il M5S, ci si può aspettare un grado di radicamento territoriale relativamente basso, in virtù della giovane età del partito e della propria struttura organizzativa. Non è tuttavia da escludere che i tentativi di radicamento territoriale del partito (Biorcio 2018) abbiano spinto, almeno in certe zone d'Italia, ad un reclutamento dei candidati all'interno degli attivisti e degli amministratori locali.

\section{ANALISI DEI DATI}

Dopo aver indicato con quali modalità abbiamo deciso di rilevare il radicamento territoriale dei candidati, passiamo adesso ad analizzare quanto siano radicati i candidati delle due diverse coalizioni.

Nella Tabella 2 sono rappresentati i dati relativi al radicamento dei candidati per coalizione o partito di appartenenza. Complessivamente, sembrerebbe essere in presenza di una distribuzione abbastanza omogenea. I dati sono infatti abbastanza equidistribuiti tra i vari valori dell'indice, con una moderata prevalenza dei candidati con profilo nazionali (il $27,8 \%$ ) e semi-nazionali (il 30,3\%), soprattutto a discapito dei candidati locali (il 17,6\%). Tuttavia, sul dato pesano in maniera decisiva i valori assunti da parte dei candidati del M5S, particolarmente differenti rispetto a quelli degli altri partiti. Più del 90\% dei candidati del Movimento (il 90,5\%) presenta

questi due limiti si è dunque scelto di disaggregare il dato solamente per la Lega e considerare gli altri candidati solo come parte della coalizione.
Tabella 2. Radicamento territoriale dei candidati nei collegi (valori percentuali, in parentesi i valori assoluti).

1 (profilo nazionale) 2 (profilo semi-nazionale) 3 (profilo semi-locale) 4 (profilo locale)

\begin{tabular}{|c|c|c|c|c|}
\hline $\begin{array}{l}\text { Coalizione di } \\
\text { centrosinistra } \\
\quad \mathrm{N}=\mathbf{2 3 1}\end{array}$ & $\begin{array}{c}14,7 \% \\
(34)\end{array}$ & $\begin{array}{c}26,4 \% \\
(61)\end{array}$ & $\begin{array}{c}35,1 \% \\
(81)\end{array}$ & $\begin{array}{c}23,8 \% \\
(55)\end{array}$ \\
\hline $\begin{array}{l}\text { Coalizione di } \\
\text { Centrodestra } \\
\quad \mathrm{N}=\mathbf{2 3 1}\end{array}$ & $\begin{array}{c}19,9 \% \\
(46)\end{array}$ & $\begin{array}{c}22,9 \% \\
(53)\end{array}$ & $\begin{array}{c}32,1 \% \\
(74)\end{array}$ & $\begin{array}{c}25,1 \% \\
(58)\end{array}$ \\
\hline $\begin{array}{c}\text { Movimento } \\
\text { Cinque Stelle } \\
\quad \mathrm{N}=\mathbf{2 3 1}\end{array}$ & $\begin{array}{l}48,9 \% \\
(113)\end{array}$ & $\begin{array}{c}41,6 \% \\
(96)\end{array}$ & $\begin{array}{c}5,6 \% \\
(13)\end{array}$ & $\begin{array}{c}3,9 \% \\
(9)\end{array}$ \\
\hline $\begin{array}{c}\text { Totale } \\
\text { candidati } \\
\mathrm{N}=693\end{array}$ & $\begin{array}{l}27,8 \% \\
(193) \\
\end{array}$ & $\begin{array}{c}30,3 \% \\
(210)\end{array}$ & $\begin{array}{l}24,2 \% \\
(168)\end{array}$ & $\begin{array}{l}17,6 \% \\
(122)\end{array}$ \\
\hline
\end{tabular}

infatti un profilo puramente nazionale o semi-nazionale, con appena il 3,9\% dei candidati con un profilo puramente locale. Valori così alti in questi due profili sono da imputare probabilmente alla mancanza di un solido radicamento territoriale del M5S e alla sua relativa novità all'interno dello scenario politico, ma anche, come abbiamo già anticipato, alle caratteristiche proprie del Movimento. La regola dei due mandati e la tendenziale impossibilità a candidarsi durante un mandato amministrativo limitano infatti la possibilità di rilevare il radicamento territoriale secondo l'indice da noi proposto.

Considerando solamente i candidati di centrosinistra e centrodestra si vede come la situazione cambi notevolmente. Il numero di candidati che possiedo un indice pari a 3 o a 4 raggiunge quasi il $60 \%$ del totale, con quasi il $25 \%$ dei candidati dei collegi con un profilo fortemente locale. Specularmente, possiamo notare come il numero dei candidati con un profilo più nazionale sia significativamente ridotto. Meno del 25\% dei candidati ha infatti un valore di radicamento pari a 2 , e complessivamente poco più di un sesto di coloro che si sono presentati nei collegi possedeva un profilo puramente "nazionale". Si è quindi in presenza di un insieme di candidati con caratteristiche simili e che, almeno in una certa misura, presentano all'atto della candidatura una qualche forma di legame con il proprio collegio di appartenenza.

Come si può vedere, non vi sono delle significative differenze tra le due coalizioni. Se il centrodestra può vantare una percentuale di candidati con un forte radicamento territoriale superiore rispetto ai suoi avversari (i candidati con un punteggio 4 rappresentano infatti il $25,1 \%$ del totale, contro il $23,8 \%$ del centrosinistra), il centrosinistra si è presentato alle elezioni con la quota minore di candidati con profilo puramente nazionale, pari al $14,7 \%$ del totale, cinque punti percentuali in meno rispetto al centrodestra (19,9\%).

A questo punto diviene interessante cercare di analizzare in che maniera siano ripartiti i candidati radicati 
Tabella 3. Radicamento territoriale dei candidati della Lega e degli altri partiti del centrodestra sul totale dei candidati della lista di appartenenza (valori percentuali, in parentesi i valori assoluti).

\begin{tabular}{|c|c|c|c|c|}
\hline & 1 & 2 & 3 & 4 \\
\hline \multirow[t]{2}{*}{$\begin{array}{l}\text { Lega } \\
N=76\end{array}$} & $13,2 \%$ & $22,4 \%$ & $30,3 \%$ & $34,2 \%$ \\
\hline & (10) & (17) & (23) & (26) \\
\hline \multirow[t]{2}{*}{$\begin{array}{l}\text { Altri centrodestra } \\
\quad \mathrm{N}=155\end{array}$} & $23,2 \%$ & $23,2 \%$ & $32,9 \%$ & $20,7 \%$ \\
\hline & (36) & $(36)$ & (51) & (32) \\
\hline
\end{tabular}

all'interno delle varie coalizioni. Se per il centrosinistra questa domanda appare poco significativa, per la sostanziale presenza di unico attore dominante sia in termini di voti sia di candidature espresse, questo non è altrettanto vero per il centrodestra. Viene da chiedersi infatti quale sia il radicamento territoriale dei candidati dei vari partiti che compongono la coalizione, in particolare, in riferimento alla Lega. Il partito di Salvini, come abbiamo visto, in passato presentava tassi di radicamento superiori a tutti gli altri partiti; rimane da chiarire, se anche dopo la svolta "nazionale", i suoi candidati presentino ugualmente indici di radicamento più alti.

Nelle tabelle che seguono sono mostrati il radicamento territoriale dei candidati della Lega e degli altri partiti della coalizione del centrodestra che in riferimento ai candidati della stessa lista sia come percentuale complessiva dei candidati aventi lo stesso indice di radicamento territoriale.

Come si vede dalla Tabella 3, i 76 candidati della Lega nei collegi presentano un radicamento territoriale significativamente più alto rispetto agli altri partiti della coalizione di centrodestra.

I due terzi dei candidati della Lega hanno infatti un indice pari o superiore a 3 , mentre gli altri partiti della coalizione si fermano a poco più del $50 \%$. Più di un terzo dei candidati poi (il $34,21 \%$ ) presenta un indice pari a 4 , quasi 15 punti in più rispetto al restante centrodestra che invece ha un valore poco superiore al $20 \%$, mentre nettamente inferiore è la percentuale dei candidati con profilo nazionale, pari al $13,2 \%$ contro il $23,2 \%$ degli altri partiti del centrodestra.

Il dato appare ancora più significativo se confrontato con il dato dei candidati del centrodestra divisi per indice di radicamento (Tabella 4).

Come si può vedere, la Lega, nonostante esprima solo 76 candidati sui 231 complessivi del centrodestra (poco più di un terzo), rappresenta all'incirca il 50\% dei candidati che ottengono un punteggio di 4 sull'indice del radicamento territoriale. Sostanzialmente quindi un candidato su due, tra quelli che si sono candidati
Tabella 4. Radicamento territoriale dei candidati della Lega e degli altri partiti del centrodestra sul totale dei candidati con stesso oindice di radicamento (valori percentuali, in parentesi i valori assoluti).

\begin{tabular}{|c|c|c|c|c|}
\hline & 1 & 2 & 3 & 4 \\
\hline \multirow[t]{2}{*}{ Lega } & $21,7 \%$ & $32,1 \%$ & $31,1 \%$ & $44,8 \%$ \\
\hline & (10) & (17) & (23) & (26) \\
\hline \multirow{2}{*}{$\begin{array}{c}\text { Altri } \\
\text { centrodestra }\end{array}$} & $78,3 \%$ & $67,9 \%$ & $68,9 \%$ & $55,2 \%$ \\
\hline & (36) & (36) & (51) & (32) \\
\hline $\begin{array}{l}\text { Totale } \\
\text { candidati }\end{array}$ & $19,9 \%$ & $22,9 \%$ & $32,1 \%$ & $25,1 \%$ \\
\hline $\mathrm{N}=231$ & (46) & (53) & (74) & (58) \\
\hline
\end{tabular}

(Fonte: Elaborazione dell'autore)

mentre ricoprivano un incarico amministrativo e sono nati all'interno del collegio uninominale di candidatura, appartiene alla Lega, percentuale significativamente più alta del numero dei candidati "nazionali" leghisti che coprono solamente il $20 \%$ del totale dei candidati del centrodestra. I candidati leghisti possiedono quindi un grado di radicamento significativamente superiore a tutte le altre forze politiche, confermandosi quindi come un partito fortemente "territoriale" in cui il legame con il territorio, ed in particolar modo l'aver ricoperto un incarico amministrativo all'interno di un ente locale, rappresenta un fattore di considerevole importanza.

Passiamo adesso ad esaminare quale sia la distribuzione geografica dei candidati in base al loro indice di radicamento territoriale. Essa infatti ci permette di comprendere se esistano delle differenze di candidatura tra candidati con un profilo maggiormente locale e altri con un profilo maggiormente nazionale. Candidati "nazionali" potrebbero infatti essere stati candidati in collegi elettorali in cui la coalizione è elettoralmente più forte al fine di poter loro garantire una rielezione relativamente "sicura". Viceversa, in collegi elettoralmente più difficili per la coalizione, potrebbe essere più vantaggioso candidare personaggi dotati di un forte radicamento territoriale al fine di risultare maggiormente competitivi.

Nelle tabelle seguenti sono riportate le ripartizioni geografiche, per forza politica, dei candidati sulla base dell'indice di radicamento posseduto, nel tentativo di evidenziare come siano suddivisi tra i collegi i candidati che possiedono un medesimo indice. La ripartizione per aree è stata fatta nella maniera seguente: nell'area Nord-Ovest sono state incluse Piemonte, Lombardia e Liguria; nell'area Nord-Est, Veneto, Friuli Venezia-Giulia e Trentino; nell'area Centro, Emilia-Romagna, Toscana, Marche, Umbria; nell'area Sud, Lazio, Abruzzo, Molise, Campania, Puglia, Basilicata, Calabria, e nell'area Isole, Sicilia e Sardegna. 
In questo caso esistono sostanziali differenze non solo con il Movimento Cinque Stelle ma anche tra le due coalizioni. Per quanto riguarda il Movimento 5 Stelle (Tabella 5) si nota come la percentuale maggiore di candidati con un profilo radicato si ha all'interno dei collegi del Nord-Ovest, dove si trovano quasi metà dei candidati aventi un punteggio pari a quattro (il 44,4\%) e del Centro Italia, dove sono candidati il 30,8\% dei candidati con un punteggio pari a tre e il $22,2 \%$ di quelli con un punteggio pari a quattro. Più omogenea la distribuzione dei candidati con profilo nazionale, con una maggiore diffusione nei collegi del Nord-Est (il 18, 6\%) e in misura minore all'interno del Sud e delle Isole, dove si trovano rispettivamente il $35,5 \%$ e il $14,6 \%$ dei candidati con profilo semi-locale. Passando adesso all'esame della coalizione di centrosinistra (Tabella 6) si nota invece come si sia in presenza di un quadro differente. La maggior parte dei candidati aventi un profilo nazionale è presente all'interno dei collegi del Centro e del Nord Italia, mentre si evidenzia una netta maggioranza di candidati con un profilo fortemente locale all'interno dei collegi del Sud Italia. Dei candidati del centrosinistra con un punteggio pari a 4, infatti, quasi uno su due (il 45,5\%) è candidato al Sud, mentre di quelli con un punteggio pari a 3 è candidato al Sud il 28,4\%. Solo il 5,9\% dei candidati con profilo nazionale sono invece stati inseriti nei collegi meridionali. Specularmente, si nota come vi sia una prevalenza di candidati nazionali nei collegi del centro Italia. Dei candidati con un profilo puramente nazionale (indice pari ad 1) il 38,1\% è stato infatti candidato all'interno di un collegio del centro, mentre solo il 9\% dei candidati del centro Italia presenta un profilo fortemente radicato all'interno del proprio collegio di riferimento.

Il dato, sebbene chiaramente preliminare e suscettibile di ulteriori approfondimenti, si presta a varie interpretazioni. Una prima ipotesi è relativa alla candidatura

Tabella 5. Distribuzione geografica dei candidati del Movimento Cinque Stelle per indice di radicamento (valori percentuali, in parentesi i valori assoluti).

\begin{tabular}{|c|c|c|c|c|}
\hline & \multicolumn{4}{|c|}{ Movimento Cinque Stelle } \\
\hline & 1 & 2 & 3 & 4 \\
\hline $\begin{array}{c}\text { Nord-ovest } \\
\mathrm{N}=60(25,9 \%)\end{array}$ & $\begin{array}{c}24,8 \% \\
(28)\end{array}$ & $\begin{array}{l}26,1 \% \\
(25)\end{array}$ & $\begin{array}{c}23,1 \% \\
(3)\end{array}$ & $\begin{array}{c}44,4 \% \\
(4)\end{array}$ \\
\hline $\begin{array}{c}\text { Nord-est } \\
\mathrm{N}=30(13,1 \%)\end{array}$ & $\begin{array}{c}18,6 \% \\
(21)\end{array}$ & $\begin{array}{c}8,3 \% \\
(8)\end{array}$ & $\begin{array}{c}7,7 \% \\
(1)\end{array}$ & $\begin{array}{l}0,0 \% \\
(0)\end{array}$ \\
\hline $\begin{array}{c}\text { Centro } \\
\mathrm{N}=40(17,3 \%)\end{array}$ & $\begin{array}{c}16,8 \% \\
(19)\end{array}$ & $\begin{array}{c}15,6 \% \\
(15)\end{array}$ & $\begin{array}{c}30,8 \% \\
(4)\end{array}$ & $\begin{array}{l}22,2 \% \\
(2)\end{array}$ \\
\hline $\begin{array}{c}\text { Sud } \\
\mathrm{N}=76(32,9 \%)\end{array}$ & $\begin{array}{c}31,0 \% \\
(35)\end{array}$ & $\begin{array}{c}35,4 \% \\
(34)\end{array}$ & $\begin{array}{c}30,8 \% \\
(4)\end{array}$ & $\begin{array}{c}33,3 \% \\
(3)\end{array}$ \\
\hline $\begin{array}{c}\text { Isole } \\
\mathrm{N}=25(10,8 \%)\end{array}$ & $\begin{array}{c}8,8 \% \\
(10)\end{array}$ & $\begin{array}{c}14,6 \% \\
(14)\end{array}$ & $\begin{array}{c}7,7 \% \\
(1)\end{array}$ & $\begin{array}{c}0,0 \% \\
(0)\end{array}$ \\
\hline $\begin{array}{l}\text { Totale } \\
\mathrm{N}=231\end{array}$ & $\begin{array}{l}48,9 \% \\
(113)\end{array}$ & $\begin{array}{c}41,1 \% \\
(96)\end{array}$ & $\begin{array}{l}5,6 \% \\
(13)\end{array}$ & $\begin{array}{c}3,9 \% \\
(9)\end{array}$ \\
\hline
\end{tabular}

(Fonte: Elaborazione dell'autore)
Tabella 6. Distribuzione geografica dei candidati della coalizione di centrosinistra per indice di radicamento (valori percentuali, in parentesi i valori assoluti).

\begin{tabular}{|c|c|c|c|c|}
\hline & \multicolumn{4}{|c|}{ Coalizione di centrosinistra } \\
\hline & 1 & 2 & 3 & 4 \\
\hline $\begin{array}{c}\text { Nord-ovest } \\
\mathrm{N}=60(25,9 \%)\end{array}$ & $\begin{array}{c}29,4 \% \\
(10)\end{array}$ & $\begin{array}{c}24,6 \% \\
(15)\end{array}$ & $\begin{array}{c}23,5 \% \\
(19)\end{array}$ & $\begin{array}{c}29,1 \% \\
(16)\end{array}$ \\
\hline $\begin{array}{c}\text { Nord-est } \\
\mathrm{N}=30(13,1 \%)\end{array}$ & $\begin{array}{c}20,6 \% \\
\text { (7) }\end{array}$ & $\begin{array}{l}8,2 \% \\
(5)\end{array}$ & $\begin{array}{c}13,6 \% \\
(11)\end{array}$ & $\begin{array}{c}12,7 \% \\
(7)\end{array}$ \\
\hline $\begin{array}{c}\text { Centro } \\
\mathrm{N}=40(17,3 \%\end{array}$ & $38,2 \%$ & $13,1 \%$ & $17,3 \%$ & $9,1 \%$ \\
\hline $\begin{array}{l}\text { Sud } \\
N=76(32,9 \%)\end{array}$ & $\begin{array}{l}5,9 \% \\
\text { (2) }\end{array}$ & $\begin{array}{c}42,6 \% \\
(26)\end{array}$ & $\begin{array}{c}28,4 \% \\
(23)\end{array}$ & $\begin{array}{l}45,5 \% \\
(25)\end{array}$ \\
\hline $\begin{array}{c}\text { Issole } \\
\mathrm{N}=25(10,8 \%)\end{array}$ & $\begin{array}{l}5,9 \% \\
(2)\end{array}$ & $\begin{array}{c}11,5 \% \\
\text { (7) }\end{array}$ & $\begin{array}{c}17,3 \% \\
(14)\end{array}$ & $\begin{array}{l}3,6 \% \\
(2)\end{array}$ \\
\hline $\begin{array}{r}\text { Totale } \\
N=231\end{array}$ & $\begin{array}{c}14,7 \% \\
(34)\end{array}$ & $\begin{array}{c}26,4 \% \\
(61)\end{array}$ & $\begin{array}{c}35,1 \% \\
(81)\end{array}$ & $\begin{array}{c}23,8 \% \\
(55)\end{array}$ \\
\hline
\end{tabular}

(Fonte: Elaborazione dell'autore)

in collegi considerati come sicuri della leadership dei partiti della coalizione di centrosinistra. Il rischio di un risultato non soddisfacente per i partiti del centrosinistra potrebbe aver spinto il gruppo dirigente del Partito Democratico e i partiti alleati a cercare candidature sicure all'interno dei collegi del centro Italia, in cui storicamente vi era una maggiore forza elettorale di questa parte politica. Una seconda ipotesi, non necessariamente in contraddizione con la prima, riguarda invece il ceto politico del centrosinistra nel contesto meridionale. La debolezza della coalizione di centrosinistra al Sud e il risultato negativo ottenuto già alle elezioni del 2013 (Chiaramonte e De Sio, 2014) potrebbero aver spinto il PD e i suoi alleati alla candidatura di amministratori locali in carica, cercando di compensare lo svantaggio competitivo attraverso la selezione di personalità particolarmente radicate all'interno dei contesti locali e con una fitta rete di relazioni e consensi personali.

Il dato del centrodestra (Tabella 7) si presenta invece come più omogeneo all'interno del territorio nazionale. Sebbene anche in questo caso si segnala una presenza maggiore dei candidati fortemente radicati all'interno dell'area meridionale (tra i candidati con indice 3 e 4 quelli candidati al Sud sono rispettivamente il 36,5\% e il 27,59\%), il dato risulta equilibrato dalla presenza di candidati fortemente radicati anche nel Nord-ovest, (rispettivamente il $31,1 \%$ e il $25,9 \%$ ). Significativa infine anche la quota dei candidati radicati nell'area centrale del Paese, con poco più del $20 \%$ dei candidati fortemente radicati che provengono da quest'area. Un certo grado di equilibrio si segnala anche in riferimento ai candidati con profilo nazionale. Salvo il caso del Nord-est e delle Isole, che pagano anche un minor numero complessivo di collegi, i candidati nazionali si distribuiscono senza scostamenti particolarmente significativi in tutte le aree della Penisola, con una quota del tutto identica nel Nord-ovest e al Sud (26,1\%). Per meglio comprendere il 
Tabella 7. Distribuzione geografica dei candidati della coalizione di centrodestra per indice di radicamento (valori percentuali, in parentesi i valori assoluti).

\begin{tabular}{|c|c|c|c|c|}
\hline & \multicolumn{4}{|c|}{ Coalizione di centrodestra } \\
\hline & 1 & 2 & 3 & 4 \\
\hline $\begin{array}{c}\text { Nord-ovest } \\
\mathrm{N}=60(25,9 \%)\end{array}$ & $\begin{array}{c}26,1 \% \\
(12)\end{array}$ & $\begin{array}{c}18,9 \% \\
(10)\end{array}$ & $\begin{array}{c}31,1 \% \\
(23)\end{array}$ & $\begin{array}{c}25,9 \% \\
(15)\end{array}$ \\
\hline $\begin{array}{c}\text { Nord-est } \\
\mathrm{N}=30(13,1 \%)\end{array}$ & $\begin{array}{c}15,2 \% \\
\text { (7) }\end{array}$ & $\begin{array}{c}11,3 \% \\
(6)\end{array}$ & $\begin{array}{c}10,8 \% \\
(8)\end{array}$ & $\begin{array}{c}15,5 \% \\
\text { (9) }\end{array}$ \\
\hline $\begin{array}{c}\text { Centro } \\
\mathrm{N}=\mathbf{4 0}(17,3 \%)\end{array}$ & $\begin{array}{c}21,7 \% \\
(10)\end{array}$ & $\begin{array}{c}13,2 \% \\
\text { (7) }\end{array}$ & $\begin{array}{c}13,5 \% \\
(10)\end{array}$ & $\begin{array}{c}22,4 \% \\
(13)\end{array}$ \\
\hline $\begin{array}{c}\text { Sud } \\
\mathrm{N}=76(32,9 \%)\end{array}$ & $\begin{array}{c}26,1 \% \\
(12)\end{array}$ & $\begin{array}{c}39,6 \% \\
(21)\end{array}$ & $\begin{array}{c}36,5 \% \\
(27)\end{array}$ & $\begin{array}{c}27,6 \% \\
(16)\end{array}$ \\
\hline $\begin{array}{c}\text { Isole } \\
\mathrm{N}=25(10,8 \%)\end{array}$ & $\begin{array}{c}10,9 \% \\
\text { (5) }\end{array}$ & $\begin{array}{c}17,0 \% \\
(9)\end{array}$ & $\begin{array}{c}8,1 \% \\
(6)\end{array}$ & $\begin{array}{c}8,6 \% \\
(5)\end{array}$ \\
\hline $\begin{array}{c}\text { Totale } \\
\mathrm{N}=231\end{array}$ & $\begin{array}{c}19,9 \% \\
(46)\end{array}$ & $\begin{array}{c}22,9 \% \\
\text { (53) }\end{array}$ & $\begin{array}{c}32,0 \% \\
(74)\end{array}$ & $\begin{array}{c}25,1 \% \\
(58)\end{array}$ \\
\hline
\end{tabular}

(Fonte: Elaborazione dell'autore)

dato del centrodestra tuttavia, è necessario però andare a disaggregarlo secondo l'appartenenza partitica dei candidati in ciascuna area geografica.

All'interno della Tabella 8 sono elencati i valori percentuali e assoluti della distribuzione geografica dei candidati della Lega e degli altri partiti di centrodestra. Come si vede, i candidati fortemente radicati della Lega sono tutti localizzati, senza significative differenze, nei collegi collocati al centro-nord del Paese. Questo è facilmente interpretabile alla luce del tradizionale insediamento territoriale del partito di Salvini.

La svolta nazionale della Lega si è prodotta solo recentemente; è lecito quindi supporre che il partito necessiti ancora di qualche anno per radicarsi nel Sud Italia. Va segnalato tuttavia che all'incirca il $20 \%$ dei candidati con un indice di radicamento pari a 3 si trova nel Sud Italia, segnale questo che può essere interpretato in maniera duplice. Da un lato, infatti, esso può essere considerato come un accenno di progressivo radica-

Tabella 7. Distribuzione geografica della Lega e degli altri partiti di centrodestra per indice di radicamento (valori percentuali, in parentesi i valori assoluti).

\begin{tabular}{|c|c|c|c|c|c|c|c|c|}
\hline & \multicolumn{4}{|c|}{ Lega } & \multicolumn{4}{|c|}{ Altri partiti del centrodestra } \\
\hline & 1 & 2 & 3 & 4 & 1 & 2 & 3 & 4 \\
\hline \multirow[t]{2}{*}{ Nord-ovest } & $30,00 \%$ & $35,29 \%$ & $39,13 \%$ & $34,62 \%$ & $25,00 \%$ & $11,11 \%$ & $27,45 \%$ & $18,75 \%$ \\
\hline & (3) & (6) & (9) & (9) & (9) & (4) & (14) & (6) \\
\hline \multirow[t]{2}{*}{ Nord-est } & $20,00 \%$ & $17,65 \%$ & $13,04 \%$ & $34,62 \%$ & $13,89 \%$ & $8,33 \%$ & $9,80 \%$ & $0,00 \%$ \\
\hline & (2) & (3) & (3) & (9) & (5) & (3) & (5) & (0) \\
\hline \multirow[t]{2}{*}{ Centro } & $50,00 \%$ & $0,00 \%$ & $26,09 \%$ & $30,77 \%$ & $13,89 \%$ & $19,44 \%$ & $7,84 \%$ & $15,63 \%$ \\
\hline & (5) & (0) & (6) & (8) & (5) & (7) & (4) & (5) \\
\hline \multirow[t]{2}{*}{ Sud } & $0,00 \%$ & $35,29 \%$ & $21,74 \%$ & $0,00 \%$ & $33,33 \%$ & $41,67 \%$ & $43,14 \%$ & $50,00 \%$ \\
\hline & (0) & (6) & (5) & (0) & (12) & (15) & (22) & (16) \\
\hline \multirow[t]{2}{*}{ Isole } & $0,00 \%$ & $11,76 \%$ & $0,00 \%$ & $0,00 \%$ & $13,89 \%$ & $19,44 \%$ & $11,76 \%$ & $15,63 \%$ \\
\hline & (0) & (2) & (0) & (0) & (5) & (7) & (6) & (5) \\
\hline Totale & $13,16 \%$ & $22,37 \%$ & $30,26 \%$ & $34,21 \%$ & $29,68 \%$ & $34,19 \%$ & $47,74 \%$ & $37,42 \%$ \\
\hline$N=231$ & (10) & (17) & (23) & (26) & (36) & (36) & (51) & (32) \\
\hline
\end{tabular}

mento del partito di Salvini nelle amministrazioni locali meridionali; dall'altro, esso può essere attribuito alla capacità della Lega di costruire una propria classe politica, laddove ne è sprovvista, attirando e candidando tra le proprie file la classe politica locale in passato legata agli altri partiti. La distribuzione delle candidature degli altri partiti del centrodestra appare invece leggermente differente con una prevalenza di candidati radicati nell'area meridionale: il $50 \%$ dei candidati con un punteggio pari a 4 e il $43 \%$ di quelli con un punteggio pari a 3 è infatti stata candidata nei collegi meridionali. Il dato che emerge dall'analisi deve tenere chiaramente conto della differente spartizione geografica dei candidati all'interno della coalizione. Infatti, com'è logico aspettarsi, la gran parte dei candidati della Lega si concentra nei collegi del Centro-Nord (dove quasi un collegio su due è riservato ad un leghista), con la naturale conseguenza di un aumento del peso delle regioni meridionali nel reclutamento dei candidati degli altri partiti del centrodestra. Tuttavia, fatte queste premesse, appare chiaro come tra le due diverse aree della coalizione emergono quindi delle significative differenze. I candidati leghisti, maggiormente radicati, sembrano provenire in gran parte dalle aree geografiche dove storicamente il partito presentava la propria maggior forza elettorale, cioè quelle del Nord-Est e del Nord-Ovest, dimostrando comunque una significativa capacità di candidare individui radicati anche all'interno di contesti di recente insediamento (come le aree del Centro-Italia) oppure addirittura di esordio (come il Sud-Italia). Dato completamente speculare quello degli altri partiti del centrodestra, che presentano un reclutamento del personale politico radicato principalmente incentrato nelle regioni meridionali del Paese, mentre proporzionalmente risultano essere più numerosi i personaggi politici di profilo nazionale candidati nelle regioni del Nord Italia.

\section{DISCUSSIONE DEI DATI}

La ricerca evidenzia come vi siano delle profonde differenze tra il M5S, in cui i candidati radicati sono in numero estremamente ridotto, e le forze politiche tradizionali, dove invece si assiste ad una forte diffusione di candidati fortemente radicati. Il M5S, come abbiamo già detto, per sua stessa natura, recluta il personale politico prevalentemente tra persone estranee allo scenario politico, limitando in questa maniera la diffusione di personalità radicate. Diverso il caso di centrodestra e centrosinistra. Relativamente a queste due coalizioni, il primo dato da segnalare è la significativa presenza di amministratori locali in carica (o appena cessati) tra 
chi è stato candidato nei collegi uninominali. In percentuali differenti, a seconda della coalizione di appartenenza, ma senza scostamenti di grandissimo rilievo, gli amministratori locali in carica sembrano rappresentare un bacino di reclutamento privilegiato per i candidati nei collegi. Le due forze politiche sembrano quindi considerare il capitale politico ( $\mathrm{ma}$ anche sociale e relazionale), che si può accumulare attraverso l'attività amministrativa, come una caratteristica fondamentale per la competizione nei collegi uninominali. In secondo luogo, bisogna sottolineare come nella diffusione dei candidati radicati all'interno dei collegi uninominali non vi siano delle significative differente tra queste due forze politiche. Il centrosinistra sembra presentare una propensione maggiore alla candidatura di personalità radicate rispetto al centro-destra, ma senza scostamenti di grandi dimensioni. Le differenze maggiori si segnalano però nella distribuzione geografica dei candidati. Il centrosinistra sembra concentrare la percentuale più alta di candidati radicati all'interno dei collegi uninominali del centro-sud, mentre ai candidati dotati di un profilo nazionale sono stati riservati i seggi del centro e del nord Italia. Come già detto, in assenza di analisi più specifiche, non si possono tirare conclusioni ma solamente avanzare ipotesi sulla ragione di questo comportamento. Ai candidati fortemente radicati sembra essere riservato il compito di aumentare la competitività della coalizione all'interno dei contesti come il Sud Italia, dove vi era una maggiore debolezza del partito e dove più alto era il rischio, poi verificatosi, di una sconfitta elettorale. Si può dunque ipotizzare che il Partito Democratico ed i suoi alleati abbiano cercato di compensare la minore capacità di competizione della coalizione all'interno di questi collegi attraverso la candidatura e i voti personali di amministratori locali candidati in queste aree.

Come abbiamo visto, anche Forza Italia ed i partiti di centrodestra ad essa alleati (Lega esclusa) presentano un andamento in qualche maniera simile a quello del centro-sinistra. Essi presentano infatti proporzionalmente un numero maggiore di candidati radicati all'interno dei collegi del Sud-Italia, mentre un numero maggiore di quelli con profilo nazionale si registra nel centro-nord. Tuttavia, le candidature del centrodestra non sembrano essere utilizzate in maniera da compensare eventuali svantaggi competitivi della coalizione. Non siamo infatti in presenza di un numero significativamente molto elevato di candidati nazionali nelle regioni del centro-Italia, dove la situazione di partenza era certamente meno favorevole alla coalizione. Il dato delle due coalizioni ci permette di aggiungere qualche ulteriore conclusione su quello che è stato il reclutamento complessivo dei candidati in questa tornata elettorale. Entrambe le coalizioni sembrano concentrare i propri sforzi nel reclutamento di personalità radicate all'interno dei collegi del Sud Italia. Il numero maggiore di amministratori candidati dalle due forze politiche proviene infatti dalle regioni meridionali del nostro Paese. Si può ipotizzare che questo comportamento sia dovuto, come già detto, alla competizione con il Movimento Cinque Stelle, attore emergente con un forte consenso al Sud, ma anche che le due maggiori coalizioni abbiano ritenuto di fondamentale importanza candidare nei collegi meridionali personalità dotate, almeno in teoria, di un insieme forte di reti e relazioni personali. Osservandone i candidati nei collegi, si può quindi dedurre che il ceto politico meridionale, espressione di queste due coalizioni, appaia fortemente legato alla presenza di legami personali e locali, secondo un modello notabilare che sembra ricordare quello della Democrazia cristiana meridionale durante la Prima Repubblica (Panebianco 1982; Caciagli 1977). Infine, i candidati leghisti si confermano per il forte legame con il territorio di appartenenza. La svolta nazionale della Lega di Salvini non sembra aver influito negativamente sul legame che tiene insieme i candidati leghisti con il territorio di appartenenza, tantoché essi presentano tassi di radicamento superiori a tutte le altre forze politiche nei territori del Nord. Il dato però significativo è la presenza di alcune personalità radicate anche all'interno dei collegi meridionali, segnale di una progressiva espansione della forza leghista anche in quelle aree, come il Meridione, dove manca un legame con il territorio di lungo periodo.

\section{CONCLUSIONI}

Sebbene rimanga da indagare l'impatto del radicamento dal punto di vista della competizione elettorale e da quello del comportamento degli eletti, i risultati della ricerca sembrano confermare gli assunti teorici di partenza che evidenziavano come il radicamento territoriale rappresentasse una dimensione importante nell'analisi del mutamento della classe politica e dei partiti. Il reclutamento dei candidati radicati sul territorio sembra avere un ruolo di primo piano nella selezione dei candidati, sebbene con modi e forme differenti da partito a partito. Con l'eccezione del Movimento Cinque Stelle, l'analisi evidenzia la presenza di un numero relativamente alto di candidati locali nei collegi, con numerosi amministratori locali candidatisi all'interno delle due coalizioni durante il proprio mandato amministrativo. Questo è vero particolarmente per le Regioni del Sud d'Italia, dove le due coalizioni hanno attinto all'insieme degli amministratori locali per cercare candidati da inserire all'interno dei collegi uninominali. Le due forze politiche più tra- 
Il radicamento territoriale dei candidati nei collegi uninominali alle elezioni politiche del 2018

dizionali sembrano dunque associare, all'interno della propria offerta politica, una forte presenza degli interessi locali all'interno della rappresentanza nazionale. Alla vigilia del prossimo referendum sulla diminuzione dei parlamentari, sarà interessante esaminare come questa presenza potrebbe evolversi in futuro in seguito ad una modifica del numero dei rappresentanti parlamentari.

Certamente questo tipo di analisi non esaurisce l'esame del radicamento territoriale. Essa infatti si è concentrata su una forma particolare di radicamento, quello relativo principalmente alle precedenti esperienze amministrative, e poco ci dice sull'esistenza di forme di radicamento territoriale di diverso tipo, anche esterne al territorio della politica (come l'aver ricoperto particolari incarichi in associazioni o imprese importanti del territorio). Infine, l'analisi, concentrandosi solamente sui candidati nei collegi alle ultime elezioni politiche, esclude una comparazione con i candidati non nei collegi e con quelli presentatisi ad altre elezioni. Tuttavia, essa apre alcune interessanti piste di ricerca sul tema. In primo luogo, emergono alcuni interessanti interrogativi sull'effettiva esistenza di un vantaggio competitivo garantito dal radicamento territoriale ai candidati radicati e alle forze politiche che li sostengono. La presenza di numerosi candidati radicati fa infatti supporre che le forze politiche ritengano elettoralmente remunerative queste candidature. E necessario dunque verificare in che maniera il radicamento incida effettivamente sui risultati elettorali. Secondariamente, la ricerca apre interessanti prospettive di analisi in chiave comparata sul ruolo del radicamento territoriale nei diversi sistemi elettorali e sui mutamenti a cui esso è soggetto in seguito a riforme della legge elettorale. Infine, essa apre alcune interessanti domande di ricerca sull'evoluzione del radicamento all'interno del sistema politico italiano. Saranno infatti necessarie ulteriori analisi volte a comprendere meglio quali saranno le trasformazioni dei partiti politici italiani sotto questo punto di vista e ad analizzare se le differenze fin qui sottolineate si manterranno anche in futuro o se invece si produrrà un progressivo processo di avvicinamento tra $\mathrm{i}$ vari attori politici. In particolare, sarà interessante verificare se il Movimento Cinque Stelle continuerà a possedere la sua propria specificità o se invece assumerà progressivamente tratti sempre più simili a quelli delle altre forze politiche, focalizzando la propria attenzione al reclutamento di personalità solidamente ancorate all'interno dei contesti locali.

\section{BIBLIOGRAFIA}

Arzheimer, K., e J. Evans 2012. «Geolocation and voting: Candidate-voter distance effects on party choiche on the 2010 general election in England.» Political Geography 31(5): 301-310.

Best, H., e Cotta M, 2003., Parliamentary Representatives in Europe 1948-2000. Oxford, Oxford University Press.

Blais, A., Gidengil, E., Dobrzynska A., Nevitte N., e Adeau R., 2003. «Does the local candidate matter? candidate effects in the canadian election of 2000.» Canadian Journal of Political Science/Reveu canadienne de science politique 36(3): 657-664.

Bogdanor, V., 1985. Representatives of the Peoples? Parliamentaries and their Constituents in Western Democracies. Gower, Aldershot,.

Borchert, J., Zeiss, Z., 2003. The Political Class in Advanced Democracy. Oxford, Oxford University Press,

Caciagli, M., 1977. Democrazia Cristiana e potere nel Mezzogiorno, Firenze, Guaraldi

Chiaramonte, A., De Sio, L., 2014. Terremoto elettorale. Le elezioni politiche del 2013. Bologna: Il Mulino,

Chiaramonte, A., D'Alimonte, R., 2018. «The new Italian electoral system and its effects on strategic coordination and disproportionality.» Italian Political 13(1): $1-11$.

Child, S, Cowely P., 2011. «The politics of local presence: is there a case for descriptive representation.» in Political studies 59(1): 1-19.

Cotta, M. 1979. Classe politica e Parlamento in Italia 1946-1979. Bologna, Il Mulino,.

Cotta, M., Best H., 2007. Democratic Representation in Europe: Diversity, Change and Convergence. Oxford: Oxford University Press.

Crisp, B. F., Desposato, S. W., 2004. «Constituency Bulding in Multimember Districts: Collusion or Conflict?» The Journal of Politics 12(66): 136-156.

D'Alimonte, R. Chiaramonte A., 1994. «Il nuovo sistema elettorale. Quali opportunità?» Rivista Italiana di Scienza Politica XXI-II(3): 513-547.

D’Alimonte, R., Bartolini S., 2002. Maggioritario finalmente? La transizione elettorale 1994-2001. Bologna, Il Mulino.

Diamanti, I., 2003.. Bianco, rosso, verde... e azzurro. Mappe e colori dell'Italia politica. Bologna, Il Mulino.

Di Virgilio, A. 1996.. «Le alleanze elettorali. Identità partitiche e logiche coalizionali.» Italian Political Science Review/Rivista Italiana di Scienza Politica 26(3): 519584.

Di Virgilio, A. 1994.. Dai partiti ai poli. la politica delle alleanze. Italian Political Science Review/Rivista Italiana di Scienza Politica 24(3): 493-547.

Dolez, B., Hastings M., 2003. Le parachutage politique, Parigi, L'Harmattan,. 
Fabbrini, S., 1996. "Luci ed ombre della transizione.» Il Mulino XLV(2): 458-467.

Fenno, R., 1978. Home style: House members in their districts. Boston. Little Brown.

Fiva, J. H., Smith, D. M., 2017.. «Local candidates and voter mobilization: Evidence from historical tworound elections in Norway.» in Electoral Studies 45: 130-140.

Fruncillo, D. e Addeo F., 2018.. Le elezioni del 2018. Partiti, candidati, regole, risultati. Firenze, SISE.

Jankowski, M., 2016. «Voting for locals: Voters' Information processing strategies in open-list pr systems.» in Electoral Studies 43: 72-84.

Lazar, M., 1988. "Communism in Western Europe in the 1980s.» Journal of Communist Studies 4(3): 243-257.

Mattina, L. 1994. "I candidati. "Italian Political Science Review/Rivista Italiana di Scienza Politica 24(3): 549586.

Mattina, L., Tonarelli, A. 1996. «I candidati. Visioni politiche e carriere.» Italian Political Science Review/Rivista Italiana di Scienza Politica 26(3): 483-517.

Marangoni, F., Tronconi, F., 2011. «When Territory Matters: Parliamentary Profiles and Legislative Behaviour in Italy (1987-2008).» The Journal of Legislative Studies 17(4): 415-434.

Marangoni, M., F. Tronconi 2009. « Looking for Locals? Electoral reforms and intraparty competition in Italy,.» Paper non pubblicato.

Melchionda, E. 2001. «L'alternanza prevista. La competizione nei collegi uninominali.» Italian Political Science Review/Rivista Italiana di Scienza Politica 31(3): 399-470.

Panebianco, A. 1982. Modelli di partito. Organizzazione e potere nei partiti politici, Bologna, Il Mulino,

Paparo, A., Emanuele V., 2018. Gli sfidanti al governo. Disincanto, nuovi conflitti e diverse strategie dietro il voto del 4 marzo 2018. Roma, Luiss University Press.

Parisi, A. M. L., Pasquino G., 1977. Continuità e mutamento elettorale in Italia. Bologna, Il Mulino.

Passarelli, G., Tuorto, D, 2018.. La Lega di Salvini. Bologna: Il Mulino.

Pitkin, H. F., 1967. The Concept of Representation. Berkeley, University of California Press,

Put, G-J., Maddens B., 2015. "The effect of municipality size and local office on the electoral success of Belgian/Flemish election candidates: a multilevel analysis.» Government and Opposition 50(4): 607-628.

Put, G-J., Smulders, J., Maddens B., 2018. «How local personal vote-eranings attributes affects the aggrate party ote share: Evidence from the Blegian flexiblelist PR system (2003-2014).» Politics.

Roy, J, Alcantara, C. 2015. "The candidate effect: does the local candidate matter?» Journal of Elections, Public Opinion and Parties 25(2): 195-214.

Russo, F., 2011. "The Constituency as a Focus of Representation: Studying the Italian Case through the Analysis of Parliamentary Questions.» The Journal of Legislative Studies 17(3): 290-301.

Shugart, M. S., Valdini, M. E, Souminen K., 2005. «Looking for Locals: Voter Information Demands an Personal Vote-Earning Attributes of Legislators under Proportional Reperesentation.» American Journal of Political Science 49(2): 437-439.

Tavits, M., 2010. «Effect of local ties on electoral success and parliamentary behaviour: The case of Estonia.» Party Politics 16(2): 215-235.

Tavits, M., 2009. «The Making of Mavericks: Local Loyalties and Party Defection.» Comparative Political Studies 42: 793-815.

Tronconi, F., Verzichelli, L., 2007. «Il ceto parlamentare alla prova della nuova riforma elettorale» In D'Alimonte, R., Bartolini S., Proporzionale ma non solo. Le elezioni politiche del 2006, a cura di, Bologna, Il Mulino, pp. 335-367.

YIN, Robert K. 2009. "How to do better case studies.» The SAGE handbook of applied social research methods, pp. 254-282.

YIN, Robert K. 2013. Validity and generalization in future case study evaluations. Evaluation 19(3): 321332.

Ventura, S., 2018. "The Italian Democratic Party from Merger to Personalism.» South European Society and Politics XXIII(1): 181-196.

Verzichelli, L., 1996. "La classe politica della transizione.» Rivista Italiana di Scienza Politica XXVI(3): 727-768.

Verzichelli, L. 2006. "La classe politica italiana, dalla crisi all'adattamento: accesso, circolazione e carriera dal 1994 al 2006.» Rivista Italiana di Scienza Politica XXXVI(3): 455-478.

Verzichelli, L., Legnante, G., 2005. «Collegio, Parlamento e comunicazione. I luoghi della rappresentanza negli orientamenti dell'élite parlamentare italiana all'epoca del (quasi) maggioritario.» Rivista Italiana di Politiche Pubbliche 3: 99-137. 